'Departamento de Imágenes. Facultad de Medicina Universidad del Desarrollo - Clínica Alemana de Santiago, Chile.

Trabajo no recibió financiamiento. La autora declara no tener conflictos de interés.

Recibido el 11 diciembre de 2018, aceptado el 14 de agosto de 2019.

Correspondencia a: Daniela Barahona Zuleta Latadia 4322, depto. 403, Las Condes, Santiago. dbarahona@alemana.cl

\section{¿Cuándo solicitar PET/CT en la evaluación de sarcoidosis?}

\author{
DANIELA BARAHONA Z. ${ }^{1}$
}

\section{When should a PET/CT be requested in sarcoidosis?}

Sarcoidosis is a multisystemic disease of variable course that mainly affects thoracic lymph nodes and lung parenchyma. However, any organ can be involved. There is no single test for the definitive diagnosis or a precise method to evaluate the course of the disease. PET/CT is not included in the standard workup for sarcoidosis, but it may assess the inflammatory activity, detect extrapulmonary disease such as cardiac involvement and evaluate the response to treatment. It is important to be aware of the optimal timing to request a PET/CT in sarcoidosis and which patients would benefit with this procedure.

(Rev Med Chile 2019; 147: 1308-1314)

Key words: Fluorodeoxyglucose F18; Positron-Emission Tomography; Sarcoidosis.
L a tomografía por emisión de positrones en conjunto con una tomografía computada logo de la glucosa marcado con flúor $\left(F D G-F^{18}\right)$ ha adquirido relevancia en la evaluación de un gran número de neoplasias, tanto en su etapificación, respuesta a tratamiento, como en la sospecha de recurrencia. Actualmente su uso se ha ampliado a la evaluación de patologías inflamatorias las cuales también muestran alto consumo de glucosa y por lo tanto son hipermetabólicas en el PET, dentro de éstas se encuentran las vasculitis de vaso grande, infecciones de etiología no precisada y la sarcoidosis.

\section{Sarcoidosis}

La sarcoidosis corresponde a una enfermedad multisistémica, dependiente de la inmunidad celular con formación de granulomas no caseificantes, de etiología desconocida ${ }^{1}$. Afecta principalmente el parénquima pulmonar y los linfáticos torácicos, pero puede comprometer cualquier órgano. Los síntomas sistémicos son comunes, tales como fatiga, sudoración nocturna y baja de peso, sin embargo, con frecuencia es asintomática o con síntomas variables dependiendo de los órganos comprometidos ${ }^{2}$.

No existe test diagnóstico definitivo, para su diagnóstico se requiere al menos de tres elementos: clínica y radiografías compatibles, exclusión de otras enfermedades y detección histológica de granulomas no caseificantes ${ }^{3}$.

El manejo de la sarcoidosis está dirigido a prevenir daño de órganos afectados, aliviar síntomas y mejorar la calidad de vida. Sin embargo, un gran número de pacientes no requiere tratamiento por presentar una enfermedad asintomática y no progresiva $^{4}$.

En el caso de la sarcoidosis pulmonar la evaluación del compromiso orgánico se realiza mediante pruebas de función pulmonar y radiografía de tórax, siendo aún utilizado el sistema de estadiaje radiográfico $^{5}$ (Tabla 1, Figura 1 ).

\section{¿Cuándo solicitar PET/CT?}

\section{Sospecha de sarcoidosis}

La evaluación con PET/CT no se recomienda en caso de sospecha de sarcoidosis. 


\section{Tabla 1. Estadiaje radiográfico de sarcoidosis}

\begin{tabular}{|ll|}
\hline Estadío 0 & Radiografía de tórax normal \\
\hline Estadío 1 & Adenopatías hiliares o mediastínicas \\
\hline Estadío 2 & $\begin{array}{l}\text { Adenopatías y enfermedad parenquimatosa } \\
\text { pulmonar }\end{array}$ \\
\hline Estadío 3 & Enfermedad parenquimatosa exclusiva \\
Estadío 4 & Fibrosis pulmonar \\
\hline
\end{tabular}

No es el estudio inicial de elección ni tampoco un método apropiado de tamizaje, principalmente por su alto costo y mayor dosis de radiación ${ }^{6}$. El estudio por imágenes inicial continúa siendo la radiografía de tórax y complementariamente, debido a su mayor capacidad de detección de las alteraciones parenquimatosas pulmonares y detección de adenopatías, la tomografía computada de tórax. El PET/CT no es capaz de diferenciar adenopatías inflamatorias de tumorales, como es el caso de pacientes con adenopatías torácicas detectadas en forma incidental, por lo cual no podrá diferenciar un paciente con linfoma y compromiso nodal de uno con adenopatías secundarias a sar- coidosis (Figura 2). No existe un punto de corte de niveles de SUV (standardized uptake value) que pueda diferenciar hipermetabolismo tumoral de hipermetabolismo inflamatorio.

En el caso de un paciente con sospecha de sarcoidosis, biopsias negativas y persistencia de la sospecha diagnóstica, el PET/CT puede aportar en la elección del mejor sitio de biopsia ${ }^{7}$, detectando las lesiones hipermetabólicas más accesibles.

\section{Evaluación de extensión de enfermedad}

El PET/CT tiene la capacidad de evaluar al paciente desde la cabeza a los pies en un solo estudio por lo cual puede detectar enfermedad oculta e inesperada en pacientes asintomáticos o con síntomas inexplicables, especialmente a nivel extratorácico (Figura 3). En un estudio retrospectivo de 89 pacientes con sarcoidosis a los cuales se les realizó PET/CT, este detectó actividad inflamatoria extrapulmonar en $80 \%$ de ellos ${ }^{8}$.

La presencia de enfermedad oculta no necesariamente llevará a la modificación de la terapia, como sería en el caso de un paciente con diagnóstico inicial de sarcoidosis sintomática. En este caso los síntomas son los que guiarán el inicio de la terapia y no necesariamente se requerirá evaluar la extensión de la enfermedad.

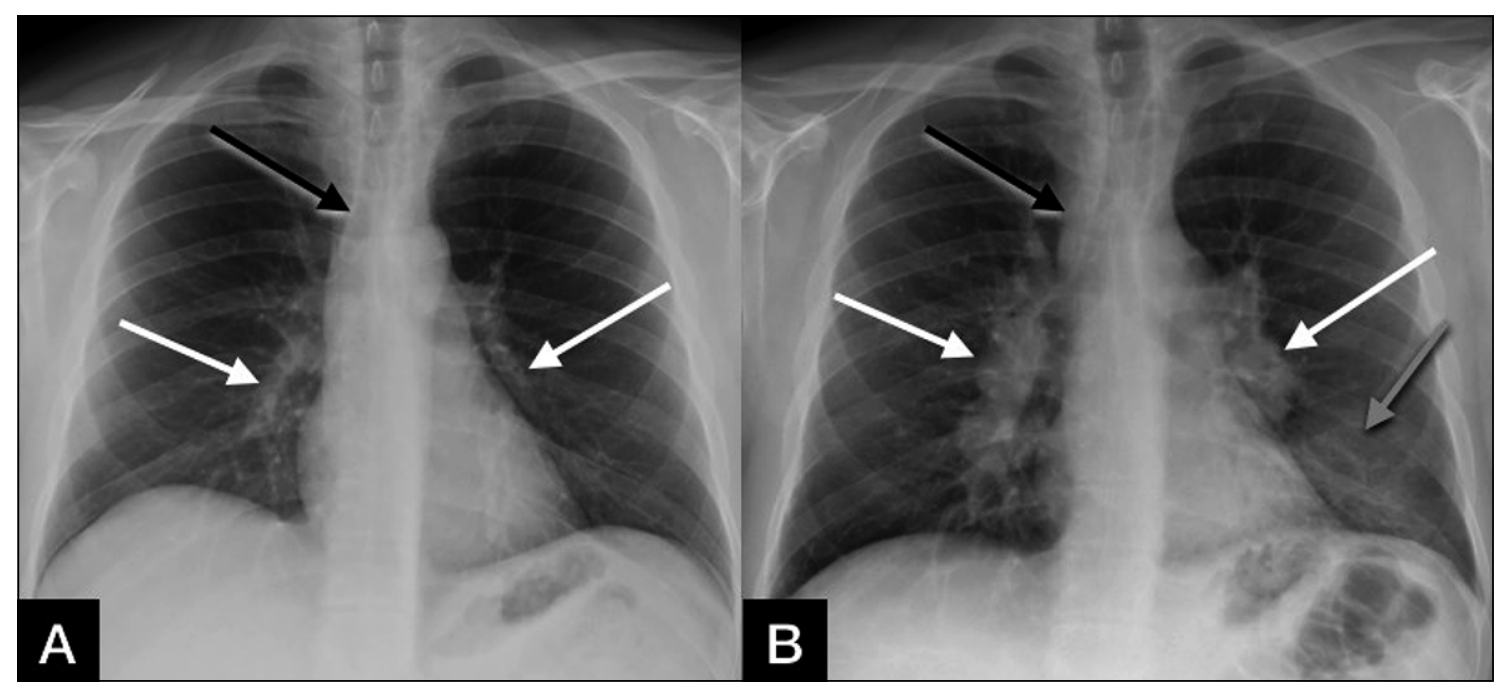

Figura 1. A. Estadío 0 de paciente con sarcoidosis, la banda paratraqueal derecha es fina (flecha negra), los hilios muestran contorno normal (flechas blancas) y no se reconocen alteraciones parenquimatosas. B. Estadío 2 del mismo paciente al año de control que muestra ensanchamiento de la banda paratraqueal secundario a adenopatías (flecha negra), convexidad de ambos hilios pulmonares (flechas blancas) y foco de condensación en el lóbulo inferior izquierdo debido a sarcoidosis alveolar (flecha gris). 


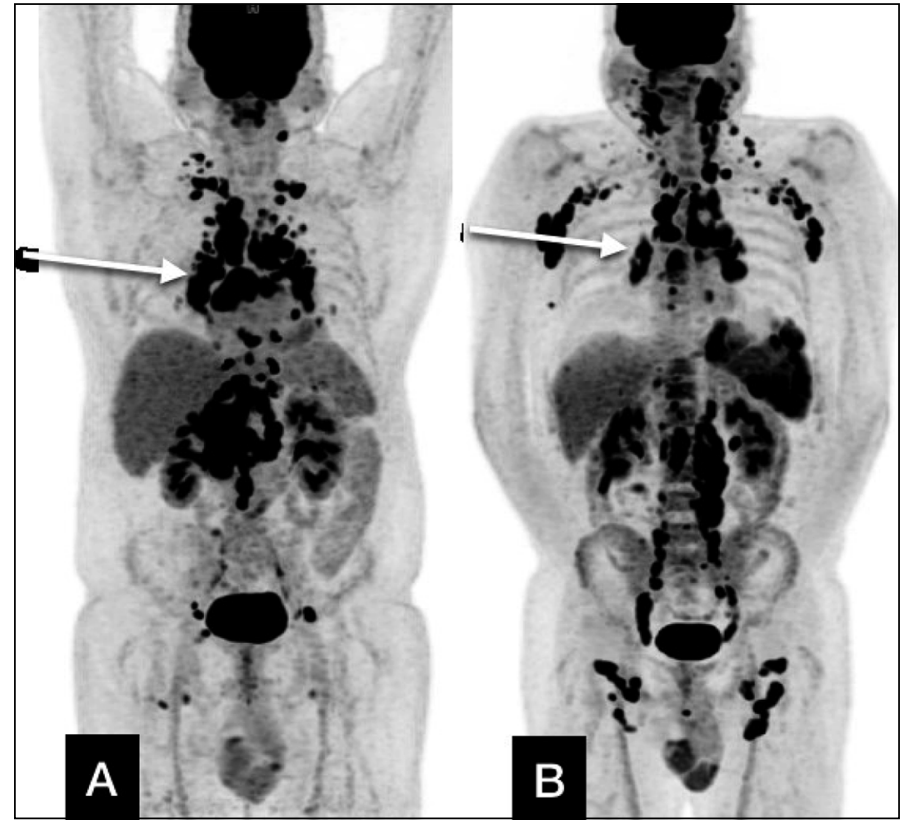

Figura 2. Imagen MIP (maximun intensity projection) de PET-FDG que demuestra innumerables adenopatías hipermetabólicas supra e infradiafragmáticas, en flechas blancas se señalan las adenopatías hiliares, la figura A corresponde a un paciente con diagnóstico de sarcoidosis y la $\mathrm{B}$ a un paciente con diagnóstico de linfoma, el grado de captación no permite diferenciar adenopatía tumoral de inflamatoria.

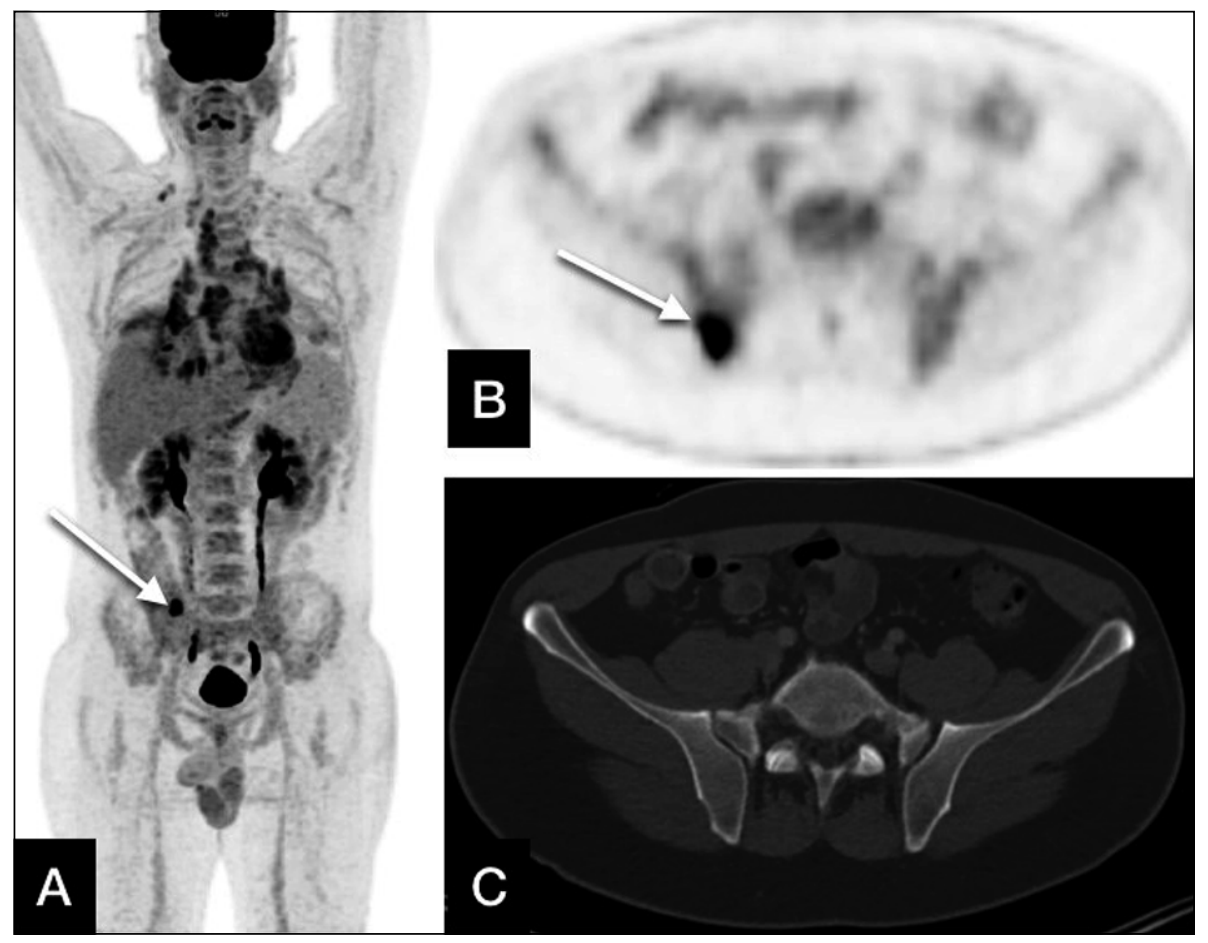

Figura 3. Paciente de 49 años con diagnóstico reciente de sarcoidosis, en A se demuestran múltiples adenopatías torácicas hipermetabólicas, algunas supraclaviculares derechas, focos hipermetabólicos pulmonares y una lesión iliaca derecha (flecha blanca en A y B) debido a sarcoidosis con compromiso óseo. En tomografía computada (figura C) no se reconoce la lesión focal ósea en la localización del foco hipermetabólico. 


\section{Evaluación de actividad inflamatoria}

El FDG/PET es un estudio sensible en la detección de actividad inflamatoria, por esto puede facilitar la distinción entre alteraciones fibróticas pulmonares, secuelas de sarcoidosis, de la enfermedad activa. El nivel de SUV corresponde a una medida semicuantitativa sin un punto de corte preciso para distinguir la presencia de actividad inflamatoria, sin embargo, la mayor captación en relación con órganos de referencia como las estructuras vasculares permite definir la positividad del estudio. En la tomografía computada la presencia de micronódulos generalmente traduce enfermedad activa, sin embargo, en pacientes con cambios fibróticos extensos la evaluación de inflamación aguda sobreagregada es dificultosa ${ }^{6}$, al igual que lo es para las pruebas funcionales (Figura 4).

Keijers et al. demostró correlación entre el metabolismo en el parénquima pulmonar con el número de neutrófilos en el lavado broncoalveolar'.

En pacientes con síntomas persistentes y marcadores serológicos negativos, el PET puede detectar inflamación activa. Algunos estudios han demostrado correlación entre la elevación de marcadores inflamatorios (neopterina, receptor soluble de interleukina 2, enzima convertidora de angiotensiva, etc) con la positividad del $\mathrm{PET}^{10}$.
Mostard et al. evaluó con PET/CT a 89 pacientes con síntomas inexplicables y persistentes demostrando lesiones activas en $73 \%$ de ellos, el $20 \%$ con marcadores serológicos negativos ${ }^{8}$.

En pacientes con diagnóstico reciente de sarcoidosis sintomática, sin duda el PET resultará positivo, por lo cual sería una herramienta innecesaria $^{11}$, sin embargo, podría aportar información en el caso de síntomas inexplicables en un paciente con compromiso extratorácico.

\section{$\underline{\text { Respuesta a tratamiento }}$}

Luego del inicio de la terapia inmunosupresora no está claro hasta cuándo mantener la terapia y no existe un método de referencia para monitorizar la respuesta a tratamiento. La respuesta usualmente se evalúa en base a síntomas, algunos test en sangre (niveles de enzima convertidora de angiotensina por ejemplo) y estudios órgano específicos como los test de función pulmonar en la sarcoidosis pulmonar, ecocardiografía en la afectación cardíaca y examen oftalmológico en el compromiso ocular ${ }^{12}$.

El PET/FDG puede ser útil en la evaluación de la respuesta, el descenso del metabolismo luego del inicio o modificación de la terapia se correlaciona con la mejoría clínica, en cambio la persistencia

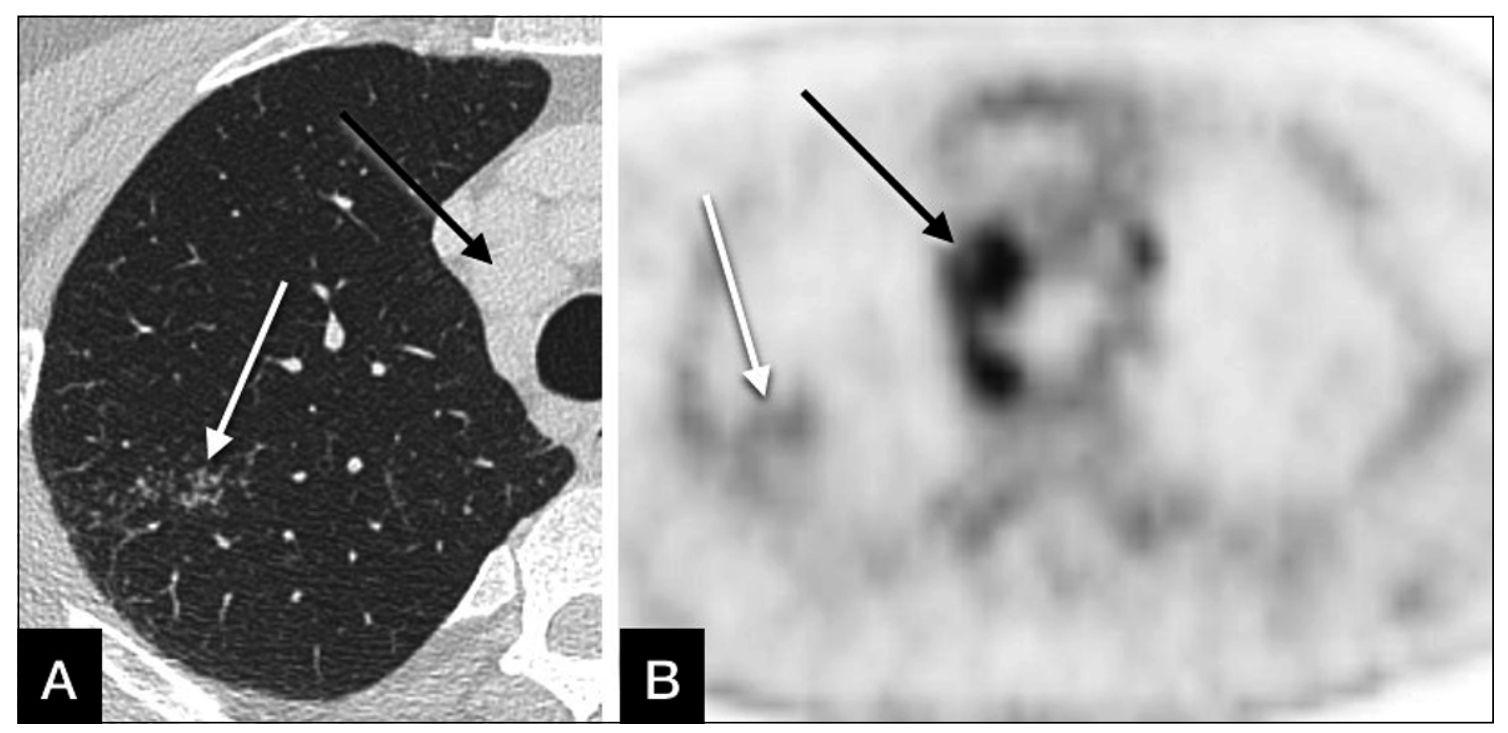

Figura 4. Paciente con sarcoidosis y micronódulos de distribución perilinfática en tomografía computada (flecha blanca en A) que se correlaciona con actividad metabólica en el PET-FDG (flecha blanca en B), además de adenopatías paratraqueales derechas hipermetabólicas (flecha negra en A y B). 


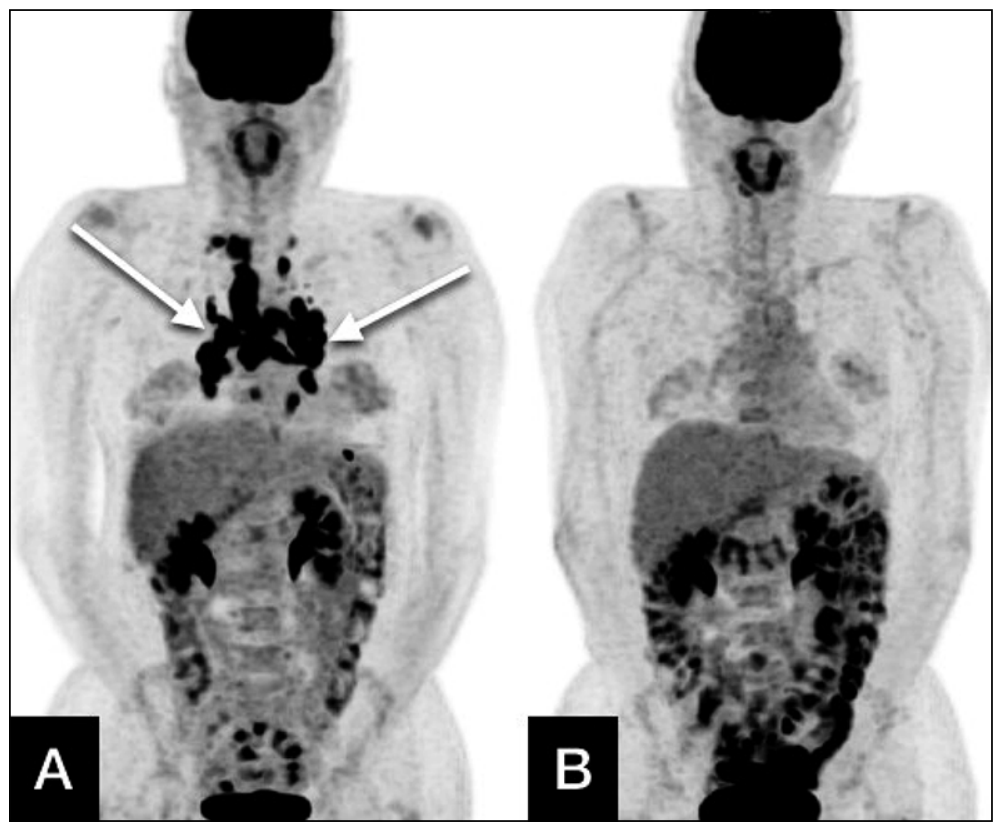

Figura 5. A. Paciente con sarcoidosis de distribución típica, con presencia de adenopatías hipermetabólicas mediastínicas e hiliares bilaterales (flechas blancas). B. Control posterior a inicio de tratamiento con corticoides que muestra respuesta metabólica completa de las adenopatías. de captación puede identificar aquellos pacientes sin una respuesta adecuada, lo que induciría el cambio de terapia ${ }^{13}$ (Figura 5).

\section{Sarcoidosis cardiaca}

Una de las causas de muerte por sarcoidosis es debida a la sarcoidosis cardiaca, siendo la evaluación del compromiso miocárdico todo un desafío $^{14}$. La afectación cardiaca reportada en Estados Unidos es de 5\%, sin embargo, en los estudios de autopsias asciende a $25 \%{ }^{15}$. Esta incidencia varía en las distintas razas, siendo más frecuente en Japón, por esto el Ministerio de Salud y Bienestar japonés cuenta con guías clínicas para la evaluación de la sarcoidosis cardiaca incorporando el uso de la resonancia magnética (RM), el PET/ CT-FDG y el holter ${ }^{16}$.

Las manifestaciones clínicas incluyen alteraciones de la conducción, arritmias, insuficiencia cardiaca congestiva y muerte súbita ${ }^{17}$. El ventrículo izquierdo es el más frecuentemente afectado ${ }^{18}$. El compromiso es típicamente parcheado por lo cual las biopsias endomiocárdicas son poco sensibles, además de ser un procedimiento invasivo.

La RM es un método sensible en la detección de la sarcoidosis cardiaca, sin embargo, puede ser dificultosa la diferenciación entre inflamación activa o fibrosis. En el caso de una RM con alteraciones cardiacas secundarias a sarcoidosis, independiente de corresponder a alteraciones activas $o$ no, el paciente puede requerir un desfibrilador o marcapasos, pero en el caso de inflamación activa, deberá además iniciar terapia inmunosupresora ${ }^{19}$.

El PET/CT es capaz de detectar inflamación miocárdica activa, sin embargo, el miocardio consume habitualmente glucosa, pudiendo ser hipermetabólico en forma difusa o parcheada fisiológicamente, lo que impide la evaluación de lesiones focales patológicas. Existen métodos para suprimir la captación miocárdica fisiológica, disminuyendo el consumo cardiaco de glucosa, ya que en condiciones de ayuno el miocardio utiliza mayormente ácidos grasos. Para lograr el consumo prioritario de ácidos grasos se indica al paciente ayuno prolongado, idealmente mayor o igual a $12 \mathrm{~h}$ y dieta baja en carbohidratos la noche anterior a la realización del examen ${ }^{20}$. Algunos estudios además incorporan la administración de heparina no fraccionada 15 min previo a la administración de FDG, ya que la heparina incrementa los niveles de ácidos grasos libres en sangre ${ }^{16}$ (Figura 6). A pesar de todas estas medidas no siempre se 


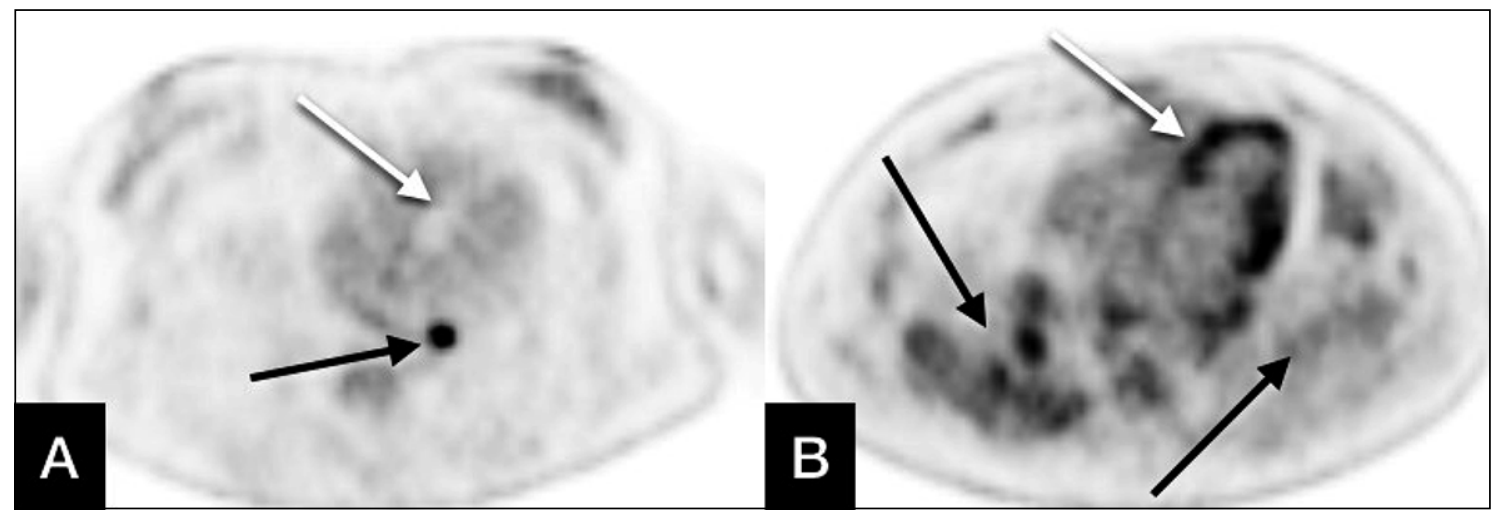

Figura 6. A. Paciente con sospecha de sarcoidosis cardiaca por resonancia magnética alterada, imágenes de PET-FDG realizadas con $12 \mathrm{~h}$ de ayuno, dieta restringida en carbohidratos el día previo y administración de heparina que logró supresión completa de la captación del miocardio (flecha blanca) sin demostrar focos hipermetabólicos que sugieran enfermedad activa. Adenopatía mediastínica hipermetabólica (flecha negra). B. Paciente con sarcoidosis pulmonar activa (flechas negras), con hipermetabolismo miocárdico difuso (flecha blanca), sin embargo este estudio no fue realizado con técnica de supresión de la captación cardiaca por lo cual no puede ser interpretado como patológico.

logra la supresión de la captación miocárdica, un metaanálisis de Youssef et al mostró sensibilidad de $89 \%$ y especificidad de $78 \%$ en el diagnóstico de sarcoidosis cardiaca ${ }^{21}$. Pueden existir falsos positivos en presencia de patología isquémica o cardiopatía hipertrófica que pueden provocar focos hipermetabólicos no inflamatorios.

\section{Conclusión}

El estudio de la sarcoidosis con PET/CT, a pesar de no formar parte del manejo rutinario, puede aportar en la valoración de la actividad inflamatoria, en la evaluación de la extensión de la enfermedad, respuesta a tratamiento, detección de enfermedad extratorácica inesperada, además de ayudar en la elección del mejor sitio de biopsia y en la evaluación de la actividad inflamatoria cardiaca.

\section{Referencias}

1. King TE. Clinical manifestations and diagnosis of sarcoidosis, 2017, UpToDate. www.uptodate.com.

2. Iannuzzi MC, Rybicki BA, Terstein AS. Sarcoidosis. N Engl J Med 2007; 357 (21): 2153-65.

3. Thomas KW, Hunninghake GW. Sarcoidosis. JAMA 2003; 289 (24):3300-3.

4. Judson MA. The treatment of pulmonary sarcoidosis.
Respir Med 2012; 106 (10): 1351-61.

5. DeRemee RA. The roentgenographic staging of sarcoidosis. Historic and contemporary perspectives. Chest 1983; 83 (1): 128-33.

6. Mostard RL, van Kroonenburgh MJ, Drent M. The role of the PET scan in the management of sarcoidosis. Curr Opin Pulm Med 2013; 19 (5): 538-44.

7. Teirstein AS, Machac J, Almeida O, Lu P, Padilla ML, Iannuzzi MC. Results of 188 whole-body fluorodeoxyglucose positron emission tomography scans in 137 patients with sarcoidosis. Chest 2007; 132 (6): 1949-53.

8. Mostard RL, Vöö S, van Kroonenburgh MJ, Verschakelen JA, Wijnen PA, Nelemans PJ, et al. Inflammatory activity assessment by F18 FDG-PET/CT in persistent symptomatic sarcoidosis. Respir Med 2011; 105 (12): 1917-24.

9. Keijsers RG, Grutters JC, van Velzen-Blad H, van den Bosch JM, Oyen WJ, Verzijlbergen FJ. (18)F-FDG PET patterns and BAL cell profiles in pulmonary sarcoidosis. Eur J Nucl Med Mol Imaging 2010; 37 (6): 1181-8.

10. Keijsers RG, Verzijlbergen FJ, Oyen WJ, van den Bosch JM, Ruven HJ, van Velzen-Blad H et al. 18F-FDG PET, genotype-corrected ACE and sIL-2R in newly diagnosed sarcoidosis. Eur J Nucl Med Mol Imaging 2009; 36 (7): 1131-7.

11. Keijsers RG, Grutters JC, Thomeer M, Du Bois RM, Van Buul MM, Lavalaye J et al. Imaging the inflammatory activity of sarcoidosis: sensitivity and interobserver agreement of (67)Ga imaging and (18)F-FDG PET. Q J Nucl Med Mol Imaging 2011; 55 (1): 66-71. 
12. Coker RK. Management strategies for pulmonary sarcoidosis. Ther Clin Risk Manag 2009; 5 (3): 575-84.

13. Sobic-Saranovic D, Grozdic I, Videnovic-Ivanov J, Vucinic-Mihailovic V, Artiko V, Saranovic D et al. The utility of 18F-FDG PET/CT for diagnosis and adjustment of therapy in patients with active chronic sarcoidosis. J Nucl Med 2012; 53 (10): 1543-9.

14. Silverman KJ, Hutchins GM, Bulkley BH. Cardiac sarcoid: a clinicopathologic study of 84 unselected patients with systemic sarcoidosis. Circulation 1978; 58 (6): 1204-11.

15. Sekiguchi M, Numao Y, Imai M, Furuie T, Mikami R. Clinical and histopathological profile of sarcoidosis of the heart and acute idiopathic myocarditis. Concepts through a study employing endomyocardial biopsy. I. Sarcoidosis. Jpn Circ J 1980; 44 (4): 249-63

16. Ishida Y, Yoshinaga K, Miyagawa M, Moroi M, Kondoh $\mathrm{C}$, Kiso $\mathrm{K}$ et al. Recommendations for 18F-fluorodeoxyglucose positron emission tomography imaging for cardiac sarcoidosis: Japanese Society of Nuclear
Cardiology Recommendations. Ann Nucl Med 2014; 28 (4): 393-403.

17. Nery PB, Beanlands RS, Nair GM, Green M, Yang J, McArdle BA et al. Atrioventricular block as the initial manifestation of cardiac sarcoidosis in middle-aged adults. J Cardiovasc Electrophysiol 2014; 25 (8): 875-81.

18. Hulten E, Aslam S, Osborne M, Abbasi S, Bittencourt MS, Blankstein R. Cardiac sarcoidosis: state of the art review. Cardiovasc Diagn Ther 2016; 6 (1): 50-63.

19. Keijsers RG, van den Heuvel DA, Grutters JC. Imaging the inflammatory activity of sarcoidosis. Eur Respir J 2013; 41 (3): 743-51.

20. Sobic-Saranovic D, Artiko V, Obradovic V. FDG PET Imaging in Sarcoidosis. Semin Nucl Med 2013; 43 (6): 404-11.

21. Youssef G, Leung E, Mylonas I, Nery P, Williams K, Wisenberg G, et al. The use of 18F-FDG PET in the diagnosis of cardiac sarcoidosis: a systematic review and metaanalysis including the Ontario experience. J Nucl Med 2012; 53 (2): 241-8. 